\title{
Characterization of ten highly polymorphic microsatellite loci for the intertidal mussel Perna perna, and cross species amplification within the genus
}

Nelson C Coelho*, Gerardo I Zardi, Gareth A Pearson, Ester A Serrão and Katy R Nicastro

\begin{abstract}
Background: The brown mussel Perna perna (Linnaeus, 1758) is a dominant constituent of intertidal communities and a strong invader with multiple non-native populations distributed around the world. In a previous study, two polymorphic microsatellite loci were developed and used to determine population-level genetic diversity in invasive and native $P$. perna populations. However, higher number of microsatellite markers are required for reliable population genetic studies.

In this context, in order to understand P. perna origins and history of invasion and to compare population genetic structure in native versus invaded areas, we developed 10 polymorphic microsatellite markers.

Findings: Described microsatellite markers were developed from an enriched genomic library. Analyses and characterization of loci using 20 individuals from a population in Western Sahara revealed on average 11 alleles per locus (range: 5-27) and mean gene diversity of 0.75 (range: 0.31 - 0.95). One primer pair revealed possible linkage disequilibrium while heterozygote deficiency was significant at four loci. Six of these markers cross-amplified in $P$. canaliculus (origin: New Zealand).
\end{abstract}

Conclusions: Developed markers will be useful in addressing a variety of questions concerning $P$. perna, including dispersal scales, genetic variation and population structure, in both native and invaded areas.

Keywords: Perna perna, Brown mussel, Genetic diversity, Invasive

\section{Findings}

The genus Perna belongs to the Mytilidae (Mollusca; Bivalvia; Lamellibranchia; Mytiloida; Mytilidae), the family of "true mussels" which includes green and brown shell mussels from tropical, subtropical, warm and cold temperate regions [1]. Species within this genus are economically and ecologically important because they constitute an important source of human food [2,3]. They are dominant species on rocky shores often forming continuous beds in the intertidal and the shallow subtidal, providing microhabitats for many species [4].

The brown mussel Perna perna is a subtropical/tropical species widely distributed along the west coast of

\footnotetext{
* Correspondence: ncoelho@ualg.pt

CCMAR, CIMAR-Laboratório Associado, University of Algarve, Campus de Gambelas, Faro 8005-139, Portugal
}

Madagascar, east African coast (from central Mozambique to False Bay), extending through the Gulf of Aden into the Red Sea, west coast of Africa (apart from the upwelling-influenced Benguela region on the west coast of South Africa) [5,6]; and from the Strait of Gibraltar to the Gulf of Tunis [7]. It is also present in Sri Lanka, southern India and in the Atlantic coast of South America where it was reported in Venezuela, Uruguay, and Brazil, as well as in the West Indies [7-9]. In Brazil, $P$. perna has been reclassified as an old introduction, most likely dating from the sixteenth century [10]. Moreover, it is reported as invasive in Western Australia [11] and in Texas, the Gulf of Mexico and southern Vera Cruz, where it was introduced via ballast water [12]. It has recently been reported for the first time on the southern 
Portuguese coast, suggesting a recent range expansion from Northern African shores [13].

Despite the economic and ecological impacts of $P$. perna, only two microsatellite markers have previously been published for this species [14]. These markers were used to score individuals from 12 populations spanning the natural and introduced ranges of the brown mussel. To improve the accuracy of genetic studies we developed and characterized additional polymorphic microsatellite markers. The microsatellite markers developed in this study will also be valuable for food forensic uses and species genetic traceability through various steps in the food chain from producer to retailers. This will enable correct identification of food varieties, which is important in order to ensure quality, safety, authenticity and health for consumers. We describe ten highly polymorphic microsatellite loci for the mussel P. perna.

Total genomic DNA was extracted from 5 individuals collected in South Africa (Port Elizabeth, 33 $58^{\prime} 47.81^{\prime \prime S}$, $25^{\circ} 39^{\prime} 30.72$ "E) using a phenol-chloroform extraction method [15]. An enriched library was made by ecogenics $\mathrm{GmbH}$ (Zurich, Switzerland) from size selected genomic DNA ligated into SNX forward/SNX reverse-linker [16] and enriched by magnetic bead selection with biotin-labeled $(\mathrm{CT})_{13},(\mathrm{GT})_{13},(\mathrm{TAC})_{10}$ and $(\mathrm{GTAT})_{7}$ oligonucleotide repeats $[17,18]$. Of 528 recombinant colonies screened, 249 gave a positive signal after hybridization. Plasmids from 96 positive clones were sequenced and primers were designed for 32 microsatellite inserts, of which 26 were tested for polymorphism. Thirteen pairs of primers were excluded because they did not amplify in at least 16 of 20 individuals initially used for polymorphism tests and three were excluded because they resulted in an allelic pattern that was difficult to interpret. The resulting ten pairs of primers were selected as polymorphic loci and were further characterized using $20 \mathrm{P}$. perna individuals from one population from Western Sahara (Boujdour, 260.'25”N, $\left.14^{\circ} 29^{\prime} 57^{\prime \prime} \mathrm{W}\right)$.

Polymerase chain reaction (PCR) was performed in volumes of $10 \mu \mathrm{l}$ containing $\pm 10 \mathrm{ng}$ of DNA, $0.5 \mu \mathrm{M}$ of each primer labelled with a florescent marker, $0.2 \mathrm{mM}$ dNTPs (Bioline), $1.5 \mathrm{mM} \mathrm{MgCl} 2,3.0 \mu \mathrm{l}$ of $5 \mathrm{x}$ PCR Buffer and $0.75 \mathrm{U}$ of GoTaq Polymerase (Promega, Madison, WI). Cycling conditions consisted of an initial denaturing step of $5 \mathrm{~min}$ at $95^{\circ} \mathrm{C}$, followed by 35 cycles of $30 \mathrm{~s}$ at $95^{\circ} \mathrm{C}, 30 \mathrm{~s}$ at annealing temperature (see Table 1 for locus optimization), $40 \mathrm{~s}$ at $72^{\circ} \mathrm{C}$, and a final elongation step at $72^{\circ} \mathrm{C}$ for 20 minutes. All PCR reactions were performed in a GeneAmp 9700 thermocycler (PE Applied

Table 1 Characterization of ten microsatellite loci for brown mussel Perna perna

\begin{tabular}{|c|c|c|c|c|c|c|c|c|c|c|}
\hline $\begin{array}{l}\text { Locus name } \\
\text { (Genebank no.) }\end{array}$ & Primer sequences & $\begin{array}{l}\text { Repeat } \\
\text { motif }\end{array}$ & $\begin{array}{l}\text { Clone } \\
\text { size (bp) }\end{array}$ & $\begin{array}{l}\text { PCR } \\
\text { annealing } \\
\left({ }^{\circ} \mathrm{C}\right)\end{array}$ & $A$ & $\begin{array}{l}\text { Size range } \\
\text { (bp) }\end{array}$ & $\mathrm{H}_{\mathrm{E}}$ & $\mathrm{H}_{\mathrm{O}}$ & $\mathrm{F}_{\mathrm{IS}}$ & $\begin{array}{l}\text { Cross } \\
\text { amplification } \\
\text { (positive/total) }\end{array}$ \\
\hline Perper-01 & F-TGGAACTTAGGGCCTTCCTC & \multirow[t]{2}{*}{$(A C)_{13}$} & \multirow[t]{2}{*}{176} & \multirow[t]{2}{*}{56} & \multirow[t]{2}{*}{9} & \multirow[t]{2}{*}{$158-183$} & \multirow[t]{2}{*}{0.8075} & \multirow[t]{2}{*}{0.45} & \multirow[t]{2}{*}{$0.46311^{* *}$} & \multirow[t]{2}{*}{$3 / 5$} \\
\hline$(J X 183697)$ & R-TCCAATTCTGTGAAATCATTGAC & & & & & & & & & \\
\hline Perper-02 & F-CCCGGTITAAGTGGTAGATG & \multirow[t]{2}{*}{$(\mathrm{GT})_{15}$} & \multirow[t]{2}{*}{113} & \multirow[t]{2}{*}{57} & \multirow[t]{2}{*}{6} & \multirow[t]{2}{*}{$110-137$} & \multirow[t]{2}{*}{0.3112} & \multirow[t]{2}{*}{0.3000} & \multirow[t]{2}{*}{0.06173} & \multirow[t]{2}{*}{$0 / 5$} \\
\hline$($ (JX183698) & R-AGCAAACGAAGGACAAATCG & & & & & & & & & \\
\hline Perper-05 & F-TCAGTGCCGCGTGATAATAC & \multirow[t]{2}{*}{$(\mathrm{TG})_{15}$} & \multirow[t]{2}{*}{127} & \multirow[t]{2}{*}{59} & \multirow[t]{2}{*}{16} & \multirow[t]{2}{*}{$111-150$} & \multirow[t]{2}{*}{0.885} & \multirow[t]{2}{*}{1.000} & \multirow[t]{2}{*}{-0.10465} & \multirow[t]{2}{*}{$4 / 5$} \\
\hline$(J X 183699)$ & R-TCCAATTACGTTTGTITTGC & & & & & & & & & \\
\hline Perper-08 & F-AATGTTCAATGTCGACAGACTATG & \multirow[t]{2}{*}{$(\mathrm{ACAG})_{7}$} & \multirow[t]{2}{*}{129} & \multirow[t]{2}{*}{56} & \multirow[t]{2}{*}{5} & \multirow[t]{2}{*}{$110-126$} & \multirow[t]{2}{*}{0.7604} & 0.5789 & $0.26394^{*}$ & $0 / 5$ \\
\hline$(J X 183700)$ & R-TTCTGAAGCACTGGATGTGG & & & & & & & & & \\
\hline Perper-11 & F-TTAACGTTGAAATCCGTGAGG & $(\mathrm{GT})_{9} \ldots$ & 133 & 58 & 16 & $70-167$ & 0.9044 & 0.4667 & $0.51000^{* *}$ & $4 / 5$ \\
\hline$(J \times 183701)$ & R-CATTCCAATCCCACGCATAC & & & & & & & & & \\
\hline Perper-16 & F-TGTGATTTAAAGTTGGACTTGTITC & $(A C)_{26}$ & 134 & 56 & 8 & $104-130$ & 0.5606 & 0.2353 & $0.60000^{* *}$ & $0 / 5$ \\
\hline$(J X 183702)$ & R-TGATTGGATCAAAATTAAACGTG & & & & & & & & & \\
\hline Perper-20 & F-ATGTCAATGTGCACAACACG & $(A C A G)_{15}$ & 245 & 55 & 27 & $202-382$ & 0.9525 & 0.9000 & 0.08065 & $0 / 5$ \\
\hline$(J \times 183703)$ & R-CGTGTATTGGCGACTIITATC & & & & & & & & & \\
\hline Perper-26 & F-CACCACCCTTACAAAGACGTG & $(A C)_{11}$ & 102 & 53 & 11 & $92-113$ & 0.845 & 0.7500 & 0.13767 & $5 / 5$ \\
\hline$(J X 183704)$ & R-TTTCACTTGGCGATTAGTATGC & & & & & & & & & \\
\hline Perper-27 & F-CCCAATTTAACGGGAACAAC & $(A C)_{27}$ & 167 & 56 & 13 & 103-193 & 0.8449 & 0.9474 & -0.09459 & $4 / 5$ \\
\hline$(J X 183705)$ & R-TTATCATCACCACTTTAAGTTACCC & & & & & & & & & \\
\hline Perper-29 & F-TTCCATITCTAGACATCTCTGTCG & $(A C)_{11}$ & 80 & 56 & 5 & $64-78$ & 0.6763 & 0.95 & -0.38314 & $5 / 5$ \\
\hline (JX183706) & R-TCAGGTGACAGCAGCCTGAC & & & & & & & & & \\
\hline
\end{tabular}

Locus name and GeneBank accession number, primer sequence, motif repetition, clone size, PCR annealing temperature, number of alleles per locus, fragment size range, gene diversity, observed heterozygosity and inbreeding coefficient (* indicates significant values at $p<0.05$ and $* *$ at $p<0.001$, after multiple tests correction). Cross amplification on $P$. canaliculus with five individuals. 
Biosystems). A DNA analyser (ABI PRISM 3130xl; Applied Biosystems) was used to analyse fragment lengths with the GeneScan Liz 500 size standard (Applied Biosystems). Raw allele sizes were scored with STRAND (http://www.vgl.ucdavis.edu/informatics/STRand), binned using the $\mathrm{R}$ package MsatAllele [19], and manually reviewed for ambiguities. Observed $\left(\mathrm{H}_{\mathrm{O}}\right)$ and expected $\left(\mathrm{H}_{\mathrm{E}}\right)$ heterozygosities were estimated, and deviations from linkage and Hardy-Weinberg equilibria were tested using GENETIX software [20]. The number of alleles per locus ranged from 5 to 27 (Table 1). Expected and observed heterozygosities ranged from 0.31 to 0.95 and from 0.23 to 1.0 , respectively, and significant heterozygote deficiency was detected in four loci after applying the q-value correction procedure (Table 1). High frequency of null alleles is likely at 3 of these loci (Perper01, Perper11 and Perper16), which was confirmed by further analysis using MICROCHECKER software [21].

We tested for linkage disequilibrium between all pairs of loci according to the procedure of Black and Krafsur [22]. The significance of the results was tested by permutation using 1000 replicates, and one pair (P20-P29) was significant at the $5 \%$ level $(\mathrm{p}=0.045)$. Although all tested individuals were heterozygous for locus Perper05 $\left(\mathrm{H}_{\mathrm{O}}=1\right.$; Table 1$)$, this result is identical to that expected under Hardy-Weinberg equilibrium $\left(\mathrm{H}_{\mathrm{E}}\right.$; Table 1$)$ for this locus, as shown by its $\mathrm{F}_{\mathrm{IS}}$ not significantly differing from zero. All markers were then tested for crossamplification in five individuals of $P$. canaliculus from New Zealand, of which several amplifications were positive (Table 1).

Microsatellite markers have been developed for other species of the genus Perna (e.g. P. canaliculus [23]; P. viridis [24]) showing levels of polymorphism similar to those of the markers described in our study. The average allele number microsatellites are 8.8 and $11.7 /$ locus for $P$. canaliculus and P. viridis respectively. Expected heterozygosity average at 0.78 and 0.69 for $P$. canaliculus and $P$. viridis respectively.

Deficiency in the number of heterozygotes observed (relative to Hardy-Weinberg expectation), with both allozyme and microsatellite studies have been documented in many marine molluscs e. g. [25-28], including species displaying separate sexes, e.g. P. perna. In this case, we cannot exclude species-specific, locus specific or population specific explanations for the results. Further genetic population structure analyses could shed light to this phenomenon in P. perna. Moreover, these ten microsatellite loci provide a useful tool to understand processes influencing species boundaries, such as range expansions outside the native distribution of $P$. perna populations, and compare diversity and differentiation scales in invasive and native populations.

\section{Availability of supporting data}

The microsatellite sequences are available through the National Center for Biotechnology Information (see http://www.ncbi.nlm.nih.gov/). The accession numbers on the repository are the following (see also Table 1):

Perper-01- JX183697; Perper-02 -JX183698; Perper-05 -JX183699; Perper-08 -JX183700; Perper-11 -JX183701; Perper-16 -JX183702; Perper-20 -JX183703; Perper-26 -JX183704; Perper-27 -JX183705 and Perper-29 -JX183706.

\section{Competing interests}

The authors declare they have no competing interests.

\section{Authors' contributions}

All authors participated in the design and implementation of the study, supervision of the work and processing interpretation of the results. CNC, NKR and ZGI participated in data analysis, microsatellite marker validation and drafted the manuscript. All authors read and approved the final manuscript.

\section{Acknowledgements}

We thank P. Blasquez for sampling, M. Valente Bernardo for sequencing and genotyping assistance. Financial support for this work was provided by Fundação para a Ciência e Tecnologia (FCT, Portugal) through a postdoctoral grant (to GIZ) and the research project PTDC/BIA-BEC/103916/2008.

Received: 5 July 2012 Accepted: 2 October 2012

Published: 8 October 2012

\section{References}

1. Gosling EM: Bivalve molluscs: biology, ecology and culture. Oxford, UK: Blackwell Science, 2003 133(5):443.

2. Griffiths $\mathrm{CL}$, Branch $\mathrm{GM}$ : The exploitation of coastal invertebrates and seaweeds in South Africa: historical trends, ecological impacts and implications for management. Trans R Soc S Afr 1997, 52:121-148.

3. Tomalin BJ, Kyle R: Subsistence and recreational mussel (perna perna) collecting in KwaZulu-Natal, south Africa: fishing mortality and precautionary management. S Afr J Zool 1998, 33:12-22.

4. Harley CDG: Climate change, keystone predation, and biodiversity loss. Science 2011, 334(6059):1124-1127.

5. Zardi G, McQuaid C, Teske P, Barker N: Unexpected genetic structure of mussel populations in south Africa: indigenous perna perna and invasive mytilus galloprovincialis. Mar Ecol Prog Ser 2007, 337:135-144.

6. Zardi G, Nicastro K, McQuaid C, Hancke L, Helmuth B: The combination of selection and dispersal helps explain genetic structure in intertidal mussels. Oecologia 2011, 165:947-958.

7. Wood AR, Apte S, MacAvoy ES, Gardner J: A molecular phylogeny of the marine mussel genus perna (bivalvia: mytilidae) based on nuclear (ITS1\&2) and mitochondrial (COI) DNA sequences. Mol Phylogenet Evol 2007, 44:685-698.

8. Vakily JM: The biology and culture of mussels of the genus perna vol. 17 . Manilla, Philippines: International Center for Living Aquatic Resources Management; 1989.

9. Berry PF: Reproduction, growth and production in the mussel, perna perna (Linnaeus), on the east coast of south Africa. In Investigational report No. 48. Edited by Institute TOR. Durban: Oceanographic Research Institute; 1978.

10. Silva EC, Barros F: Macrofauna bentonica introduzida no brasil: lista de especies marinhas e dulcıcolas e distribuiçao atual. Oecologia Australis 2011, 15:326-344.

11. Hayes K, Sliwa S, Migus F, McEnnulty, Dunstan PK: National priority pests. Parkes, Canberra, Australia: Australian Government Department of the Environment and Heritage; 2005.

12. Hicks DW, Tunnell JWJ: Invasion of the south Texas coast by the edible brown mussel perna perna (Linnaeus, 1758). Veliger 1993, 36:92-99. 
13. Lourenço C, Nicastro KR, Serrão EA, Zardi GI: First record of the brown mussel (perna perna) from the European Atlantic coast. Marine Biodiversity Record 2012, 5:e39.

14. Holland BS: Invasion without a bottleneck: microsatellite variation in natural and invasive populations of the brown mussel perna perna (L). Marine Biotechnol 2001, 3(5):407-415.

15. Nicastro K, Zardi G, McQuaid C, Teske P, Barker N: Coastal topography drives genetic structure in marine mussels. Mar Ecol Prog Ser 2008, 368:189-195.

16. Hamilton MB, Pincus EL, Di Fiore A, Fleischer RC: Universal linker and ligation procedures for construction if genomic DNA libraries enriched for microsatellites. Biotechniques 1999, 27:500-507.

17. Gautschi B, Tenzer I, Muller JP, Schmid B: Isolation and characterization of microsatellite loci in the bearded vulture (gypaetus barbatus) and crossamplification in three old world vulture species. Mol Ecol 2000, 9(12):2193-2195

18. Gautschi B, Widmer A, Koella J: Isolation and characterization of microsatellite loci in the dice snake (Natrix tessellata). Mol Ecol 2000, 9(12):2192-2193.

19. Alberto F: MsatAllele_1.0: An R package to visualize the binning of microsatellite alleles. J Hered 2009, 100(3):394-397.

20. Belkhir K, Borsa P, Chikhi L, Raufaste N, Bonhomme F: GENETIX 4.05, logiciel sous Windows TM pour la génétique des populations. Montpellier, France: Laboratoire Génome, Populations, Interactions, CNRS UMR 5000, Université de Montpellier II; 1996. 1996-2004.

21. Van Oosterhout C, Hutchinson WF, Wills DPM, Shipley P: Micro-checker: software for identifying and correcting genotyping errors in microsatellite data. Molecular Ecology Notes 2004, 4(3):535-538.

22. Black WC, Krafsur ES: A FORTRAN program for the calculation and analysis of two-locus linkage disequilibrium coefficients. TAG Theor Appl Genet 1985, 70(5):491-496.

23. MacAvoy ES, Wood AR, Gardner JPA: Development and evaluation of microsatellite markers for identification of individual greenshell ${ }^{\mathrm{TM}}$ mussels (perna canaliculus) in a selective breeding programme. Aquaculture 2008, 274:41-48.

24. Lin G, Feng F, Yue GH: Isolation and characterization of polymorphic microsatellites from Asian green mussel (perna viridis). Molecular Ecology Notes 2007, 7(6):1036-1038.

25. Zouros E, Foltz DW: Possible explanation of heterozygote deficiency in bivalve molluscs. Malacologia 1984, 25:583-591.

26. Raymond M, Vaeaentoe RL, Thomas F, Rousset F, de Meeues T, Renaud F: Heterozygote deficiency in the mussel mytilus edulis species complex revisited. Mar Ecol Prog Ser 1997, 156:225-237.

27. Borsa P, Zainuri M, Delay B: Heterozygote deficiency and population structure in the bivalve ruditapes decussatus. Heredity 1991, 66:1-8.

28. Andrade SCS, Solferini VN: Fine-scale genetic structure overrides macroscale structure in a marine snail: nonrandom recruitment, demographic events or selection? Biol J Linn Soc 2007, 91:23-26.

doi:10.1186/1756-0500-5-558

Cite this article as: Coelho et al: Characterization of ten highly polymorphic microsatellite loci for the intertidal mussel Perna perna, and cross species amplification within the genus. BMC Research Notes 2012 5:558.

\section{Submit your next manuscript to BioMed Central and take full advantage of:}

- Convenient online submission

- Thorough peer review

- No space constraints or color figure charges

- Immediate publication on acceptance

- Inclusion in PubMed, CAS, Scopus and Google Scholar

- Research which is freely available for redistribution

Submit your manuscript at www.biomedcentral.com/submit

C Bïomed Central 